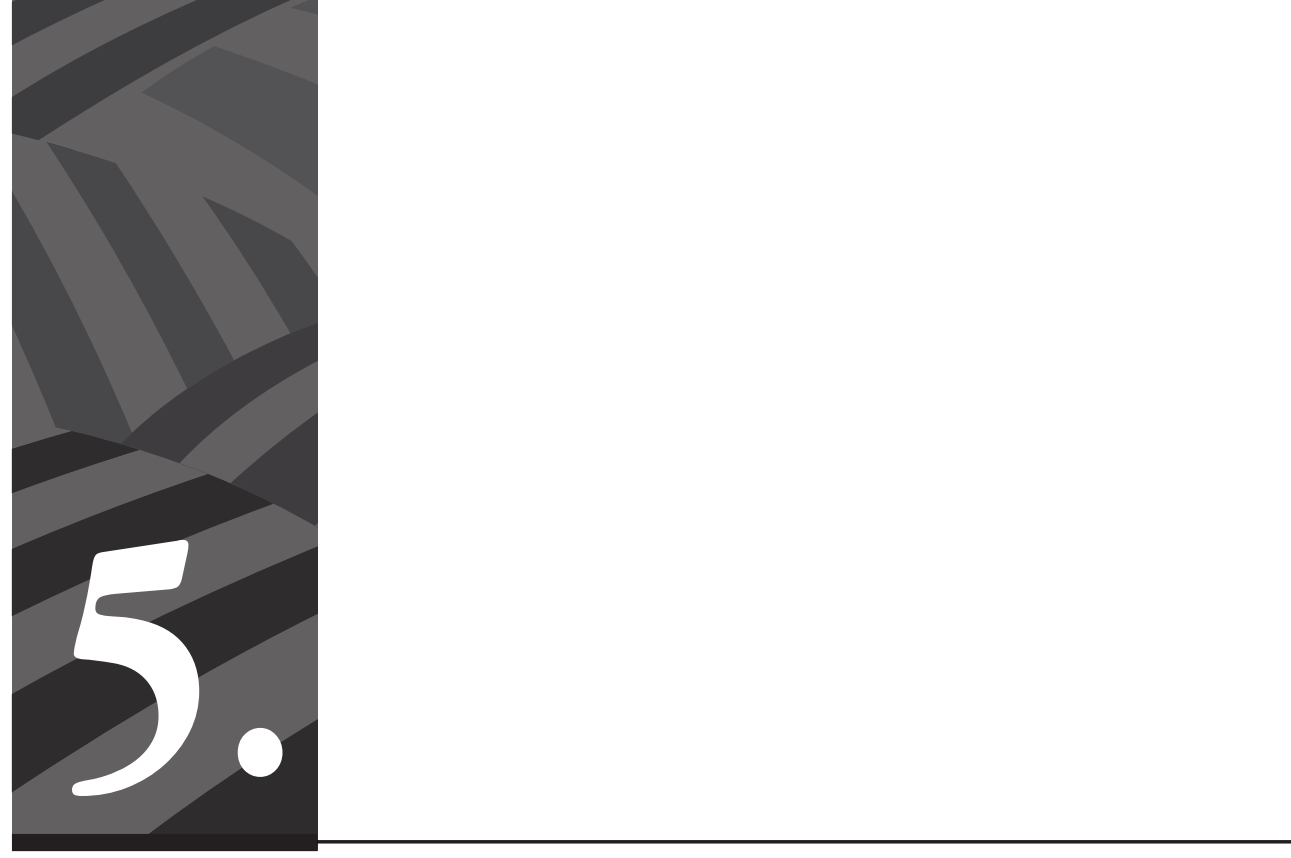

Perpetuación del despojo: Petróleo e inseguridad jurídica en la tenencia de la tierra en el Caquetá 


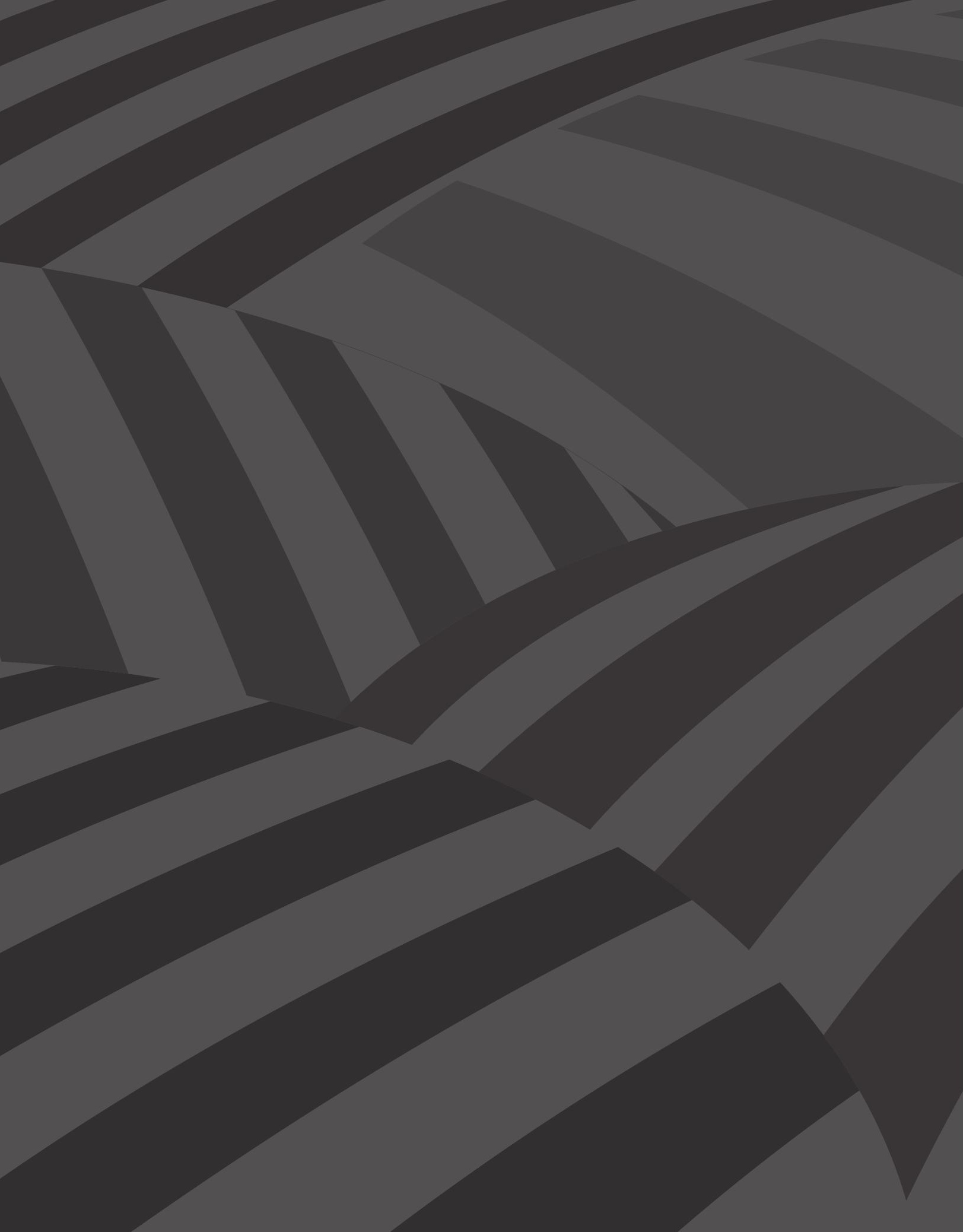




\section{Perpetuación del despojo: Petróleo e inseguridad jurídica en la tenencia de la tierra en el Caquetá}

Por Karla Díaz Parra*

Resumen: La estructura de tenencia de la tierra en el Caquetá se caracteriza por una marcada diferencia entre grandes y pequeños propietarios y una alta informalidad en la tenencia. El conflicto armado en el departamento, particularmente a partir de la retoma militar del Caguán (2002-2010), ha reforzado estas características. Sobre este mismo territorio, desde el año 2006, se inicia el proceso de adjudicación de bloques petroleros.

Este artículo se pregunta por la incidencia de la adjudicación de bloques petroleros y las posibilidades de retorno de las víctimas de desplazamiento que, al salir de sus predios, no contaban con títulos de propiedad. Sostengo que la industria petrolera se ha servido de la informalidad en la tenencia y el desplazamiento para recrear la ficción de un territorio vacío en donde desarrollar sus actividades extractivas. Este análisis recoge los aportes teóricos de David Harvey (2004) sobre acumulación por desposesión, el estudio del mercado de tierra de Karl Polanyi (2007) y Saskia Sassen (2010) sobre el nuevo orden institucional privado.

Palabras clave: vaciamiento, acumulación, tenencia de la tierra, petróleo, desplazamiento.

\section{Perpetuation of dispossession: Oil and legal insecurity in land tenure in Caquetá}

Abstract: The land tenure structure in Caquetá is characterized by a marked difference between large and small landowners and a high informality in tenure. The armed conflict in the Department, particularly since the military takeover of Caguán (2002-2010), has reinforced these characteristics. On the same territory since 2006 the process of awarding oil blocks.

This article asks about the incidence of the allocation of oil blocks and the prospect to return for victims of displacement who, when they leave their land, didn't had land deeds. I argue that the oil industry has used informality in tenure and displacement to create the fiction of an empty territory in which to develop its extractive activities.

* Politóloga de la Universidad del Rosario y Magíster en Desarrollo Regional del Colegio de la Frontera Norte en México. Correo electrónico: karladiazpa@gmail.com 
This analysis includes the theoretical contributions of David Harvey (2004) on accumulation by dispossession, the study of the land market by Karl Polanyi (2007) and Saskia Sassen (2010) about the new private institutional order.

Keywords: emptying, accumulation, land tenure, oil, displacement.

Cómo citar este artículo: Díaz Parra, Karla (2019). Perpetuación del despojo: Petróleo e inseguridad jurídica en la tenencia de la tierra en el Caquetá. Revista Controversia, 212, 143-174.

Fecha de recepción: 10 de diciembre de 2018

Fecha de aprobación: 12 de febrero de 2019

\section{Introducción}

$\mathrm{E}$ 1 departamento del Caquetá se caracteriza por una alta informalidad en la tenencia de la tierra, la cual es definida por la Organización de las Naciones Unidas para la Alimentación y la Agricultura (FAO, por sus siglas en inglés) (2003) como la ausencia de reconocimiento de los derechos de propiedad y su protección oficial. En el caso del departamento del Caquetá, la Unidad de Planificación Rural Agropecuaria (UPRA) (2017) ha calculado un índice de informalidad en la tenencia de $57.4 \%{ }^{1}$.

La definición de informalidad como ausencia de reconocimiento oficial, implica en sí misma la imposibilidad de un conocimiento veraz sobre la situación jurídica de la propiedad en el país, más aún si esta se encuentra en zonas rurales. Nelson Neva (2014) ha señalado como dificultades para el cálculo de la informalidad 1) la ausencia de un inventario sobre los predios baldíos; 2) la desactualización de la base catastral; 3) la inadecuada relación entre la información de la Superintendencia

1 La UPRA calcula el índice de informalidad a partir de la Base Catastral del IGAC, la Interrelación Catastro - Registro (ICARE) y los registros de falsa tradición ante la Superintendencia de Notariado y Registro (SNR). 
de Notariado y Registro (SNR) y la información catastral²; 4) así como la ausencia de conocimiento por parte de la población sobre los procedimientos de formalización.

En el departamento del Caquetá la situación de informalidad se ve agudizada por la presencia de la Zona de Reserva Forestal que ocupa el $56.287 \%$ del territorio (Corpoamazonía, 2012) y la presencia de 13 áreas protegidas total o parcialmente dentro del departamento. Entre ellas cabe resaltar los Parques Nacionales Naturales (PNN) Cordillera de los Picachos (82 648 ha), Serranía del Chiribiquete (3 203281 ha), Alto Fragua Indiguasi (76 $118 \mathrm{ha}$ ), Cueva de los Guacharos (1147 ha) ${ }^{3}$ (RUNAP, s.f.).

Estas figuras de protección ambiental no permiten la ocupación y uso de tipo agropecuario, situación que agudiza la informalidad, pues en realidad amplias zonas bajo figuras de protección ambiental se encuentran ocupadas (Gómez, 2017; SINCHI, 2011; Díaz, 2018).

Sumado a lo anterior, el Centro Nacional de Memoria Histórica (CNMH, 2017) ha señalado como causa de la informalidad de la tenencia de la tierra en el Caquetá, la existencia de otros ordenes jurídicos según los cuales el mercado de tierra es «regulado por las juntas de acción comunal y por los grupos armados ilegales, lo que permite realizar transacciones de compraventa de tierras al margen de las regulaciones estatales» (p. 160).

2 El documento CONPES 3641 del 2010 establece la Política nacional para Consolidar la Interrelación entre Catastro y Registro. No obstante, hasta la fecha no existe tal interrelación, lo que se evidencia en los estudios más actuales realizados por el Centro Nacional de Memoria Histórica (CNMH, 2017) y la UPRA sobre informalidad en la tenencia.

3 Las demás áreas son: Cerro Páramo de Miraflores Rigoberto Urriado, corredor Biológico Guácharos Purcé, Cuenca del río Ceibas, El Arruyo, Hacienda Esmeraldas, Hacienda Villa Mery, Áreas Naturales La Siberia y parte de la cuenca alta del río Ceibas, Miraflores Picachos. 
Además de la alta informalidad en la tenencia de la tierra en el Caquetá, en el periodo 2002- 2014 se presentó, en este departamento, una tendencia de aumento en el índice de concentración de la propiedad en pocas manos (Gini de propietarios), con un pico en los años 2013-2014 de 0.89 ; 0.15 puntos porcentuales por encima de la media nacional (0.73) (UPRA, 2015).

Al mismo tiempo se presentaron los más altos índices de desplazamiento y despojo de tierra en el departamento, debido al aumento de confrontaciones armadas durante el proceso de retoma del Caguán entre 2002 y 2007 (CNMH, 2017). El más alto número de desplazamiento se produjo en el año 2002 con 31294 personas (Unidad de Atención y Reparación Integral a las Victimas, 2018) y el mayor número de despojos se produjo en el año 2007 con 350 registros de abandono (CNMH, 2017).

En este escenario de alta informalidad, y una tendencia de concentración de la propiedad rural, se otorgan las primeras adjudicaciones de bloques petroleros. El primero de ellos fue el bloque Ombú en el municipio de San Vicente del Caguán en el año 2006 (Contrato de Exploración y Producción -E\&P- 043 ${ }^{4}$ ). A la fecha, en el Caquetá hacen presencia 9 empresas, 25 bloques petroleros han sido adjudicados, dentro de los cuales 22 se encuentran en procesos de exploración y 1 en producción.

Para el presidente Iván Duque

la industria de los hidrocarburos ha sido vital en la historia de desarrollo de nuestro país (...) Por eso he dicho, durante tanto tiempo, que lo que Colombia necesita es entender que hay que saber aprovechar la riqueza del subsuelo para derrotar la pobreza del suelo. (Presidencia de la República, 2018)

Como consecuencia, en el próximo cuatrienio se propone consolidar el sector minero energético, teniendo como meta pasar de 46 a 207

4 Los contratos E\&P son una modalidad de contratación para la exploración y producción de hidrocarburos. 
pozos exploratorios y acelerar los procesos de adjudicación de bloques disponibles (Departamento Nacional de Planeación, 2019). En el caso del departamento del Caquetá, se encuentran disponibles 15 bloques petroleros para ser adjudicados.

En este contexto surge la pregunta acerca de la incidencia de la adjudicación de bloques petroleros en el Caquetá desde el año 2006 hasta la fecha, sobre el acceso y la restitución de tierras a personas que, al ser desplazadas, no tenían títulos de propiedad. Esta es, en definitiva, una pregunta sobre las garantías de restitución y no repetición para las víctimas de conflicto en un escenario de informalidad y violencia. Para analizar las posibles tensiones que surgen en este encuentro, se analiza la relación entre informalidad, desplazamiento forzado y adjudicaciones petroleras en el Caquetá.

Como hipótesis se sostiene que la informalidad en la tenencia de la tierra y los altos índices de desplazamiento en el Caquetá han llevado a una falta de reconocimiento de los predios abandonados, creando la ficción de espacios vacíos que, al ser ocupados por la industria petrolera, niegan la posibilidad de retorno a la población desplazada.

Algunas conclusiones de esta investigación permiten afirmar que la política de mercado rural, planteada por la Ley 160 de 1994, ha funcionado como un catalizador de la informalidad y de la desigualdad en la tenencia de la tierra en el Caquetá. Por su parte, la estrategia de retoma militar de la zona de despeje (2002-2010), en el marco de la política de Seguridad Democrática, profundizó la concentración de la propiedad y repercutió en la ausencia de reconocimiento de los predios abandonados.

Finalmente, la ocupación de estos espacios por parte de empresas petroleras estuvo acompañada por un andamiaje normativo que ha otorgado garantías contractuales y de seguridad física a la actividad extractiva. El resultado es la perpetuación de las condiciones de informalidad y violencia, vista en términos de imposibilidad de retorno de población desplazada, mientras se expanden las zonas disponibles para la actividad petrolera. 


\section{Estructura del documento}

Para analizar la incidencia de la adjudicación petrolera en el Caquetá es necesario reconstruir dos momentos: en primer lugar, el proceso histórico de configuración de la estructura de tenencia de la tierra en el departamento y; en segundo lugar, la extracción de petróleo como nueva apuesta para el desarrollo y sus implicaciones en términos de reconocimiento de los predios abandonados.

En el primer caso, se analizan brevemente los procesos de asentamiento, apropiación y ordenamiento del suelo durante la colonización, para luego analizar los efectos de la implementación de la Ley 160 de 1994 sobre las dinámicas de acceso y formalización de la propiedad rural bajo el modelo de mercado de tierras en el Caquetá.

Con el fin de comprender las dinámicas de ocupación del territorio y su relación con los auges extractivos, se acude a las investigaciones de Artunduaga (1987), Cubides (1998) y Reyes (2009). El análisis de la política rural, la informalidad y la relación con élites locales se sirve de los estudios de Balcázar, López, Orosco y Vega (2001), Mondragón (2001) y Ciro (2013).

Para evidenciar el impacto en términos de formalidad y concentración de la propiedad de la tierra, a partir de la entrada en vigor de la política de mercado de tierras (Ley 160 de 1994) en el Caquetá, se recopilaron datos del Instituto Colombiano de Desarrollo Rural (Incoder) para el periodo 2002-2014, cifras de la UPRA y los datos recopilados por el CNMH en el informe Caquetá: "la tierra no basta". Para el análisis de estos datos se acoge la perspectiva de Karl Polanyi (2007) sobre el mercado de tierras y los estudios del Observatorio de Restitución y Regulación de Derechos de Propiedad Agraria ${ }^{5}$.

5 El Observatorio está conformado por la Universidad Nacional de Colombia, Universidad del Norte, Universidad Sergio Arboleda, Universidad del Rosario y Universidad del Sinú. 
En el segundo caso, se analiza la reconfiguración del estado ${ }^{6}$ en función de la apertura de la Amazonía a la extracción petrolera, analizando la correspondencia entre la puesta en marcha de los procesos de retoma militar (2002-2010), el auge del desplazamiento y la adjudicación de bloques petroleros. Al respecto, se presentan algunos testimonios sobre la informalidad en la tenencia y concentración de la propiedad rural y su relación con el conflicto armado entre los años 2002 y 2010. Las entrevistas fueron realizadas a líderes de organizaciones de desplazados entre los años 2014 y 2015 en los municipios de Florencia, Montañita y Cartagena del Chairá.

Así mismo, se acude a información de la Agencia Nacional de Hidrocarburos (ANH) y al análisis realizado por Sassen (2010) en torno al orden institucional privado, y el planteamiento de Harvey (2004) acerca de la acumulación por desposesión. En este apartado se retoman algunos testimonios de población residente en San Vicente del Caguán, San José del Fragua y Solita, zonas donde se han adelantado procesos de exploración de hidrocarburos.

\section{Luchas por la tierra: entre el petróleo y el reconocimiento}

\section{Ocupación desregulada: informalidad y acumulación}

Con respecto a las dinámicas de ocupación del Caquetá, Artunduaga (1987) relata los procesos de asentamiento en el piedemonte amazónico que surgen con el auge extractivo de la quina, desde 1890 hasta el auge del caucho en 1930. En este periodo, conocido como la «colonización espontánea», se empieza a configurar un escenario caracterizado por la ocupación desordenada e irregular de predios, ausencia de vías y bienes básicos, así como el inicio de una etapa de acumulación de tierras sin regulación por parte de empresarios caucheros.

6 En este artículo se escribe “estado” sin mayúscula acogiendo la propuesta de Ingrid Bolívar (2011), a partir de la cual el estado se entiende como un proceso de construcción socio-histórico en los territorios y no como un ente totalizante y homogéneo. 
Con el conflicto colombo-peruano y la construcción de obras de infraestructura para la guerra, principalmente vías de acceso, se produjo un nuevo fenómeno migratorio compuesto por trabajadores y militares que se asentarían en el territorio luego de finalizado el conflicto (Cubides, 1998). La ausencia de obras de infraestructura y servicios públicos fue una de las constantes en los procesos de colonización. Sin embargo, esta ausencia sirvió como catalizador de la construcción de lazos de solidaridad entre los recién llegados, quienes conformaron juntas de colonos para coordinar labores de construcción de obras como puentes, vías, centro de salud y escuelas (Moreno, 2015).

El rápido crecimiento y expansión de las zonas de colonización, y su función como refugio para los campesinos desplazados del centro del país con el estallido de la violencia, implicó mayor presión por la tierra en las zonas de «frontera» ${ }^{7}$ (Serje, 2011); esto condujo a la necesidad de una intervención más decidida por parte del estado. Como respuesta, se crearon los proyectos de fortalecimiento a la colonización ${ }^{8}$ Caquetá I (1963-1971), financiado por USAID y el proyecto de Colonización del Caquetá (1971-1975 y 1976-1980), financiado por el Banco Mundial (Martínez, 2016).

En el marco de estos proyectos, el Instituto Colombiano de Reforma Agraria (Incora) desarrolló programas de construcción de vías, crédito agropecuario y fomento de la actividad ganadera. Según informes del proyecto Caquetá I, entre 1963 y 1966 se destinaron \$117 000 000, de los cuales el $80 \%$ se destinó para la actividad ganadera y el 20 \% para agricultura (Hormaza, 2016, p. 104).

7 Serje presenta el término de frontera como una construcción ideológica con la que se han designado los territorios lejanos al centro de poder. Según la autora, los territorios de frontera han sido designados como territorios salvajes que debían ser dominados e incluidos a la economía nacional, bajo modalidades de sobreexplotación de la naturaleza y las personas.

8 Los primeros proyectos de colonización dirigida fueron desarrollados por la Caja Agraria en 1959. El Incora en los proyectos de colonización buscó dar garantías para la permanencia de las personas que ya se habían asentado en el territorio hasta la década de 1960 (Hormaza, 2016, p. 98). 
Las élites locales, muchas de ellas oriundas del departamento del Huila, promovieron y aprovecharon la política ganadera para ampliar sus propiedades y hacerse a los favores e incentivos del estado (Ciro, 2013). Por esta razón, la legalización y titulación de predios, así como la construcción de bienes rurales favorecieron principalmente a los grandes hacendados (Mondragón, 2001).

Con respecto a los proyectos de colonización adelantados por el Incora desde 1962, Mora (s.f.) plantea que estos

reproducen (sic) la estructura agraria del interior del país, en lugar de contribuir a establecer un esquema más equitativo de distribución de la tierra. Este hecho refuerza la tendencia concentradora que provoca la consolidación de la colonización, las condiciones de pobreza de los colonos iniciales y las técnicas de "tumbe y quema" aplicadas en la incorporación de tierras baldías determinan en buena parte los tamaños de propiedad”. (Hormaza, 2016, p. 141)

Por tanto, la acción del estado para dar respuesta a la creciente informalidad en la tenencia y la inequitativa distribución de la tierra durante el siglo XX fue marginal. Gran parte de los beneficios de los proyectos de reforma rural fueron cooptados por los intereses locales implicados en la expansión indebida de predios, en la mayoría de los casos, por medios violentos (Belalcázar et al., 2001). El estado tampoco pudo garantizar la permanencia de los campesinos en las parcelas que el Incora tituló, ampliando por esta vía la frontera agrícola (Rodríguez, 2013).

\section{Mercado de tierras}

«El impacto del neoliberalismo debe entenderse en el contexto de las estructuras sociales (agrarias) pre-existentes y del marco institucional en el que se adoptó» (Peña, Parada y Zuleta, 2014, p. 163). De ahí la importancia de retomar los procesos de colonización espontánea que surgen a raíz de los auges económicos y la violencia, así como los proyectos de colonización dirigida por el estado desde 1962 en cabeza del Incora. 
En este caso, la liberalización de la economía desde inicios de los años 90 y su aplicación en la política rural, a partir del modelo de mercado de tierras introducido por la Ley 160 de 1994, se ubica en un escenario de incapacidad institucional para formalizar la propiedad rural y garantizar la distribución equitativa de los beneficios de los proyectos de reforma rural.

La Ley 160 de 1994 abandonó las apuestas de una reforma rural y transitó hacia un modelo de compra y venta de tierra para la productividad ${ }^{9}$. Este modelo convirtió al estado en un intermediario de las transacciones comerciales sobre la tierra. Su papel en términos de redistribución de la propiedad se concentró en la adjudicación de baldíos y el subsidio de un 70 \% del valor comercial de la tierra para la adquisición de predios, en caso de población sin tierra.

Según Peña et al. (2014) el «diseño institucional contenido en las normas jurídicas sobre propiedad agraria, en el período 1991-2010 no tiene como fundamento la distribución de la tierra, y que, por el contrario, incentiva o permite la concentración de la misma» (p. 140). En consonancia, Balcázar, López, Orozco y Vega (2001) manifiestan que

En cinco años de funcionamiento del mercado de tierras (1995-1999) las tierras que ingresan al Fondo Nacional Agrario disminuyen a 286.939 hectáreas, mientras que el número de familias beneficiadas baja a 19.397, en comparación con el periodo anterior. La inversión cae, el crédito complementario al subsidio para la adquisición de tierras no funcionó en concordancia con aquél; el sistema de reforma agraria no funcionó como tal debido a la formulación aislada de políticas y prioridades propias en cada entidad, al grado disímil en la planificación de instituciones y entidades territoriales, a la especialización y dispersión de funciones y al estado diferenciado del proceso de descentralización en todas ellas. (p. 18)

9 Este modelo atiende a las medidas de ajuste estructural promovidas por el Fondo Monetario Internacional y el Banco Mundial como medidas para asegurar el pago de la deuda externa (Millet y Toussaint, 2005). 
En el caso del departamento del Caquetá, a pesar de que la Ley 160 de 1994 prohibió la adjudicación de más de 450 hectáreas, «las (sic) adjudicaciones de más de 1.000 hectáreas representaron sólo el 0,56 \% del total de adjudicaciones, pero llegaron a controlar el 16,54 \% del total de superficie adjudicada» (CNMH, 2017, p. 137). Jhon Freddy Vásquez, funcionario del Incoder, manifiesta: «La acumulación de tierras acá es un problema grave, porque hay muchas personas con latifundios, por ejemplo, en la sabana de San Vicente, que es de los Pozos hacia adentro, hay personas que pueden llegar a tener 2000 y 5000 hectáreas» (comunicación personal, 18 de febrero del 2014).

De igual forma, se produjo una reducción significativa de las dinámicas de titulación de baldíos en el Caquetá, «pasando de 8.602 adjudicaciones en el período intercensal 1973-1985 a 2.100 en el período intercensal 1993-2005» (CNMH, 2017, p. 139), una reducción de más del 75 \%. Esto lleva a pensar que el modelo de mercado de tierras, lejos de favorecer la formalización de la propiedad rural y controlar la concentración, ha profundizado estos fenómenos gracias a la reducción de las dinámicas de titulación de baldíos ${ }^{10}$ y el crecimiento de la gran propiedad.

Karl Polanyi (2007), en su estudio La gran transformación. Crítica del liberalismo económico, analiza el surgimiento del modelo de mercado de tierras y sostiene que la hipótesis del mercado regulado no es más que una ficción hecha a medida de los intereses de las empresas. La figura del mercado regulado parte de premisas falsas como la del homo economicus y el mercado como estructura económica básica de las culturas, según las cuales el hombre por naturaleza busca maximizar sus ganancias, aunque ello pueda ir en detrimento del bienestar de otros.

Estas premisas son desvirtuadas por el autor a partir de una revisión histórica del comportamiento económico de diferentes culturas, en donde

10 Gómez (2017) afirma que: "La estructura de tenencia de la tierra para el municipio de Florencia en su mayoría es informal, es decir, los campesinos no cuentan con títulos de propiedad que les brinden seguridad jurídica de la posesión de sus predios, producto especialmente de la baja titulación de baldíos” (p. 6). 
se evidencia que las formas más tempranas de intercambio estuvieron marcadas por lógicas comunitarias y no capitalistas, lo cual lleva a pensar que los presupuestos básicos de la economía de mercado son el resultado del modelaje que las estructuras de poder hacen sobre ella.

Ahora bien, la ficción del mercado regulado se sirve de otra falacia: la idea de la tierra como mercancía. Al respecto, Polanyi afirma que la tierra no puede concebirse como un bien, pues no es producida por el hombre, ni puede ser realmente apropiada por este. Por tal razón, la conversión de la tierra en una mercancía es una ficción creada a la medida de los intereses del capital que requiere de su usurpación.

Particularmente en el caso del Caquetá, la ley de tierras entra en funcionamiento en un espacio marcado por la informalidad en los derechos de propiedad; una gran asimetría de poder entre élites políticas y campesinos sin tierra. En este escenario, la figura del mercado de tierras afianzó el poder terrateniente e hizo posible la apertura de nuevos espacios para posar el capital extranjero en forma de explotación petrolera, promesa de eficiencia en el uso de la tierra.

\section{Violencia, despojo e informalidad}

Luego del fracaso de los diálogos de paz entre el expresidente Andrés Pastrana (1998-2002) y la guerrilla de las Fuerzas Armadas Revolucionarias de Colombia-Ejército del Pueblo (FARC-EP) ${ }^{11}$, fue electo el expresidente Álvaro Uribe Vélez (2002-2006) (2006-2010), cuya bandera principal fue la retoma de la zona de despeje y la salida militar al conflicto armado. Faivre (2005) señala que la estrategia de retoma puso en operación en el Caquetá tres batallones móviles de carabineros, 16 Batallones de mi Pueblo y 18 mil nuevos soldados en el territorio, además de la Fuerza de Tarea Conjunta Omega, la cual mezclaba tácticas de guerra por aire, mar y agua.

Según Ramírez (2009), González (2012), CODHES (2011) y CNMH (2017), el proceso de retoma militar provocó un aumento de desplazamiento,

11 Ahora partido político Fuerza Alternativa Revolucionaria del Común. 
civiles muertos en combate, violaciones de derechos humanos por parte de miembros de la fuerza pública y el involucramiento de la sociedad civil en el conflicto armado, a partir de estrategias de cooperación cívicomilitar en acciones bélicas.

El CNMH (2017) señala que en el proceso de retoma, particularmente entre los años 2002-2008, se desató una estrategia de

Copamiento militar y operaciones de tierra arrasada: (...) cuando se hicieron intensos bombardeos y el Ejército nacional realizó despliegues rápidos en los caseríos de las zonas rurales. En esa época se dieron desplazamientos masivos en la Unión Peneya, en el municipio de La Montañita, y en Peñas Coloradas, en Cartagena del Chairá, debido a la llegada del Ejército a estos caseríos. También se dieron capturas masivas en zonas rurales de San Vicente del Caguán, Puerto Rico y Cartagena del Chairá (p. 194).

\section{Gráfica 1. Desplazamiento e índice Gini de propietarios ${ }^{12}$ en el Caquetá}

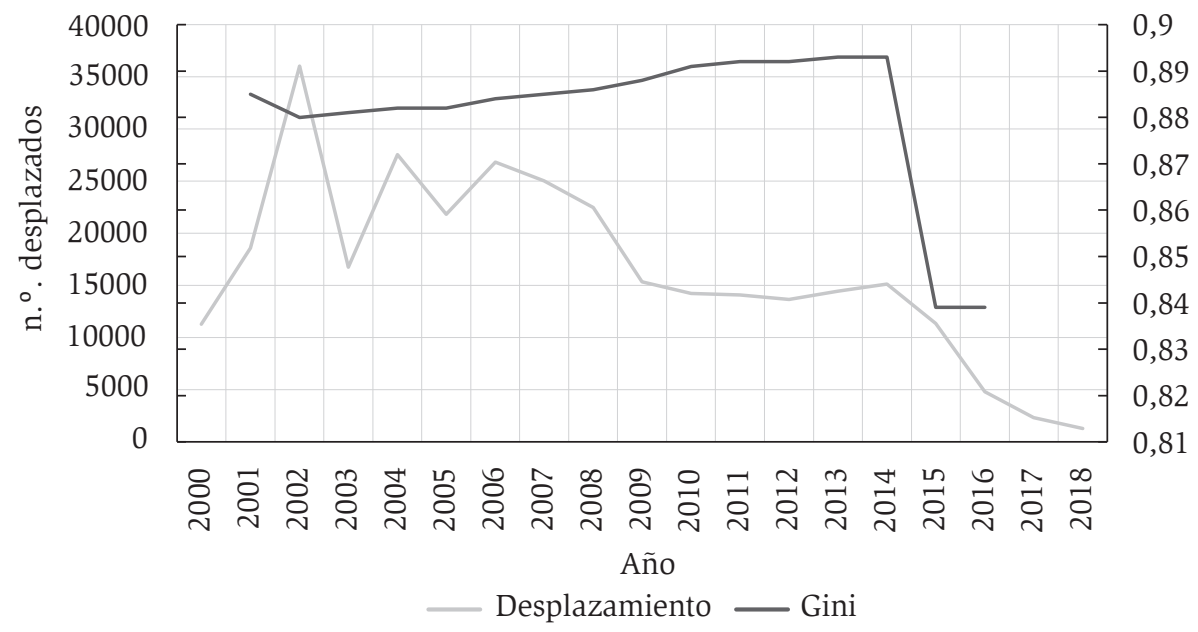

Fuente: IGAC (2014) y Unidad para la Atención y Reparación Integral a las Víctimas (2018).

12 El índice de Gini de propietarios calcula el número de hectáreas concentradas por una persona, ponderada por el número de propiedades de esta. 
Como se evidencia en la gráfica 1 , durante la retoma militar se produce un aumento en el número de personas desplazadas, así como un aumento en el índice de Gini de propietarios, lo que conduce a afirmar que existe un proceso paralelo de despojo y acumulación de tierra.

En efecto, entre el año 2002 y 2018 la cantidad de desplazados ascendió a 228544 personas registradas, con un pico en el año 2002 con un saldo de 31229 personas despojadas (UARIV, 2018). Ahora bien, existe un gran margen de no denuncia de desplazamiento y subregistro de predios despojados.

Ana María Lizcano, desplazada del municipio de Cartagena del Chairá, ante la pregunta de si había declarado su condición de desplazamiento dijo: «No, la declaración como desplazada yo lo hice apenas este año (2014), porque pues cuando nosotros salimos para aquí pa Montaña no sabíamos que uno podía hacer esa declaración de desplazamiento». Frente a la pregunta de si había regresado a su finca respondió: «Sí, y el problema que vi es que esa tierra ya la tienen otras personas y yo ya no sé qué hacer» (comunicación personal, 15 de diciembre del 2014).

En el Caquetá no es posible conocer con certeza el número de personas desplazadas y menos aún las dinámicas de despojo, puesto que

la inexistencia de un proceso sistemático de despojo (sic) se da también por una imposibilidad fáctica, ya que muchos de los predios del Caquetá no tienen títulos de propiedad y es mucho más complicado desarrollar mecanismos de despojo formal a partir de compraventas a bajo precio o por presión, como en la zona norte del país, donde existe una estructura agraria más formalizada. Las tipologías jurídicas del despojo son un poco más difíciles de identificar en el Caquetá, pues allí se dan casos de tipologías materiales de abandono, sin que pueda afirmarse que la nueva persona que ocupa el predio lo hace de mala fe o en connivencia con algún actor armado. (CNMH, 2017, p. 230) 
Por tanto, las dinámicas de despojo son subestimadas gracias a la alta informalidad en la tenencia de la tierra. Esta situación se convierte en una oportunidad para la acumulación de predios. Esta hipótesis es confirmada cuando el CNMH (2017) afirma que el aumento de las compraventas entre 2004 y 2012 coincidió con los picos de desplazamiento y de abandono, los cuales se relacionan con el fin del proceso de negociación y el inicio de la retoma, así como la puesta en marcha de la Política Nacional de Consolidación Territorial (PNCT) ${ }^{13}$. Esto indicaría, a su vez, un proceso de negociación sobre los predios abandonados (pp. 213-214).

Al respecto, Jesús Benavides, desplazado en el año 2007 de Remolinos del Caguán, municipio de Cartagena del Chairá zona priorizada de la PNCT, expresa

No, pues por ejemplo yo, yo sé que a mí ya no me devuelven la tierra, tuve un inconveniente grave y no fue por culpa mía, por culpa del Estado también, porque el Estado se adueñó de mi tierra en el 2006 cuando el señor Uribe puso la situación por allá muy dura y ese fue el problema, que a mí me dañaron la casa, el gobierno se adueñó de la casa por 15 días [...] Allá vive un Hacendario, él fue el que se quedó con todo el terreno y él no lo entrega. Él es dueño de muchas tierras por allá, él las compró muy baratas en un tiempo [...] Él quedó con un montón de tierra y eso ya no hay nada, yo decir que a mí me devuelven la tierra, no creo, uno ponerse a alegar con ese señor es muy verraco, porque tiene muchas influencias dentro del stado. (comunicación personal, 15 de diciembre del 2014, p. 36)

En definitiva, la arremetida del estado contra las FARC-EP tuvo una gran repercusión en las dinámicas de desplazamiento y concentración

13 La PCNT tuvo por objetivo controlar el territorio nacional en pugna por la presencia de grupos armados y los cultivos de uso ilícito, para luego estabilizar la presencia de actores estatales, especialmente la fuerza policial, y así consolidar estas zonas por medio de la presencia de instituciones del estado (FIP, 2011). 
de la propiedad. Desde la perspectiva acumulación por desposesión planteada por David Harvey (2004), la informalidad en la tenencia y el desplazamiento masivo funcionan como mecanismos para desocupar espacios, donde luego se pueden deslocalizar excedentes de capital, en forma de inversión extranjera.

Según Harvey (2004) la crisis del capitalismo se expresa en las contradicciones de la sobreacumulación, pues la sobreoferta de trabajo conlleva al desempleo y los excedentes de capital llevan a la devaluación de la moneda, la existencia de mercancías que no se venden y la capacidad productiva inutilizada. Para burlar el colapso del modelo, el capitalismo ha avanzado hacia la ocupación de nuevos territorios y el desplazamiento temporal de las inversiones (proyectos a largo plazo).

La ocupación de nuevos territorios se realiza por medio de la deslocalización de capitales, mejor conocido como inversión extranjera. Esta deslocalización implica la creación de condiciones para la reproducción de las lógicas del capital, en muchos casos de forma violenta.

El capital, en su proceso de expansión geográfica y desplazamiento temporal que resuelve las crisis de sobreacumulación a la que es proclive, crea necesariamente un paisaje físico a su propia imagen y semejanza en un momento, para destruirlo luego. Esta es la historia de la destrucción creativa (con todas sus consecuencias sociales y ambientales negativas) inscripta en la evolución del paisaje físico y social del capitalismo. (Harvey, 2004, p. 4)

En el caso del Caquetá, los espacios del despojo son asumidos por el estado como territorios baldíos que pueden ser ocupados por la industria petrolera. Esta imagen es reproducida en el discurso y en la cartografía nacional, pues en ellos no se muestran los terrenos ocupados no titulados ni los despojados. Así se explica cómo, en este escenario de violencia, informalidad y acaparamiento, se incrusta la política minero-energética, la cual viene a ocupar los espacios vaciados por el conflicto armado. 


\section{La política petrolera en el Caquetá y la legalización del despojo}

El municipio de San Vicente del Caguán ha sido un lugar emblemático como escenario del conflicto armado, particularmente durante el proceso de retoma y la implementación de la PNCT. Paradójicamente, de símbolo de guerra pasó a convertirse en el primer municipio en el que se otorgó un contrato de Exploración y Producción (E\&P) de hidrocarburos en el departamento ${ }^{14}$.

Para comprender esta convergencia es necesario partir de relacionar las estrategias militares de retoma y las políticas de seguridad inversionista promovida por el gobierno del expresidente Álvaro Uribe Vélez. En efecto, la PNCT buscó

Promover la seguridad como generadora de crecimiento económico y rentabilidad social. Las operaciones de la Fuerza Pública deben seguir contribuyendo a generar un clima de confianza y estabilidad que atraiga la inversión privada, produzca crecimiento económico y desarrollo social a través de la reducción del desempleo y la pobreza. Una mayor seguridad debe generar las condiciones para el adecuado desempeño de la acción social del Estado en beneficio de los más necesitados (FIP, 2011, p. 26).

La seguridad física de la infraestructura petrolera y su movilización por las carreteras del país fue una prioridad para el Gobierno nacional desde la Política de Consolidación de la Seguridad Democrática y luego la PNCT, lo que se consolidó en el departamento del Caquetá en la creación del Batallón Especial Energético y Vial n. ${ }^{\circ} 19$ entre los municipios de San Vicente del Caguán y Puerto Rico.

14 En el siglo XX se realizaron actividades de exploración de hidrocarburos en el Caquetá por parte de las empresas Texas Petroleum Company, Shell Petroleum Company y Roosvelt Oil and Gas (Arcila, 2011, p. 64). 
Garantizar la reproducción del capital deslocalizado requiere del apoyo de las instituciones financieras y gubernamentales (Harvey, 2004). En el caso de la inversión extranjera en el sector de hidrocarburos se brindaron garantías de protección física y beneficios económicos. De ahí que la política de seguridad democrática y el incentivo a la inversión extranjero, pilares del gobierno de Álvaro Uribe Vélez (2002-2006) (2006-2010), vengan juntos.

La apertura del mercado nacional a la inversión extranjera, su exaltación como principal fuente de desarrollo económico, la concesión expedita de permisos y licencias ambientales, la flexibilización de los criterios ambientales y las gabelas tributarias ${ }^{15}$ concedidas al sector minero-energético son muestra de un nuevo andamiaje que blinda de seguridad contractual y física a la actividad extractiva (Garavito, Iregui y Ramírez, 2012).

Fernando Vargas (2013), en su estudio Minería, conflicto armado y despojo de tierras: Impactos, desafíos y posibles soluciones jurídicas, presenta la convergencia entre las zonas de retoma militar y las zonas aptas para la extracción de minerales. Así se explica la correspondencia entre los espacios despoblados por la acción militar y las zonas hoy aptas para ser adjudicadas a la actividad petrolera. Para Harvey (2004) este es el modo de operación del capital transnacional para deslocalizar sus actividades y ocupar espacios en países como Colombia.

En el caso del Caquetá, los procesos de vaciamiento no solo se evidencian en la protección física de las empresas, sino también en la reacomodación de las cartografías y las culturas (formas de producción y apropiación del territorio). Desde el año 2010, el Gobierno nacional ha realizado cambios en la cartografía nacional y las políticas públicas

15 Al respecto ver el último estudio de Pardo y Duarte (2018) sobre los beneficios tributarios al sector minero- energético y el detrimento que ello ha significado para las finanzas nacionales. 
orientadas al piedemonte amazónico con el fin de viabilizar la explotación de hidrocarburos.

El Plan Nacional de Desarrollo 2010-2014 del gobierno de Juan Manuel Santos excluyó al piedemonte amazónico de la Amazonía, mediante la creación de la Región Sur como región administrativa para la destinación de recursos del Sistema General de Participaciones (SGP) ${ }^{16}$. Posteriormente, el Plan Nacional de Desarrollo 2014-2018 consolidó la política extractiva como motor de desarrollo del Caquetá, al eliminar la Región Amazonía y crear en su lugar la Región Centro-Sur y Región Llanos.

Para el gobierno de Juan Manuel Santos, el piedemonte amazónico «es una zona predominantemente montañosa con importantes desarrollos agropecuarios y recursos minero-energéticos. En el futuro, esta franja será un punto estratégico para el país en materia de conectividad» (DNP, 2014, p. 634). Así, las estrategias de política desde el gobierno central para el piedemonte se enfocan en el aprovechamiento (exploración y producción) de la naturaleza del subsuelo.

Este cambio en la cartografía y en el lenguaje con el cual se orientan los enfoques de política pública territoriales, no solo tuvo repercusiones en el aumento de las adjudicaciones de bloques petroleros, sino también en las identidades de los pobladores de estos territorios.

Guillermo Peña, expresidente de la Asociación de Juntas de San Vicente del Caguán, expresa al respecto: «A nosotros nos cambiaron de Región Amazónica a Región Centro Sur, para hacerle las cosas más fáciles a las empresas, aquí hay títulos mineros por doquier, no importa ríos, quebradas y caños, nada» (comunicación personal, 9 de enero del 2015).

16 Esta nueva regionalización se halla en las bases del PND 2010-2014. La regionalización en los PND funciona como un mecanismo para la formulación de políticas públicas y destinación de recursos con enfoque territorial. 


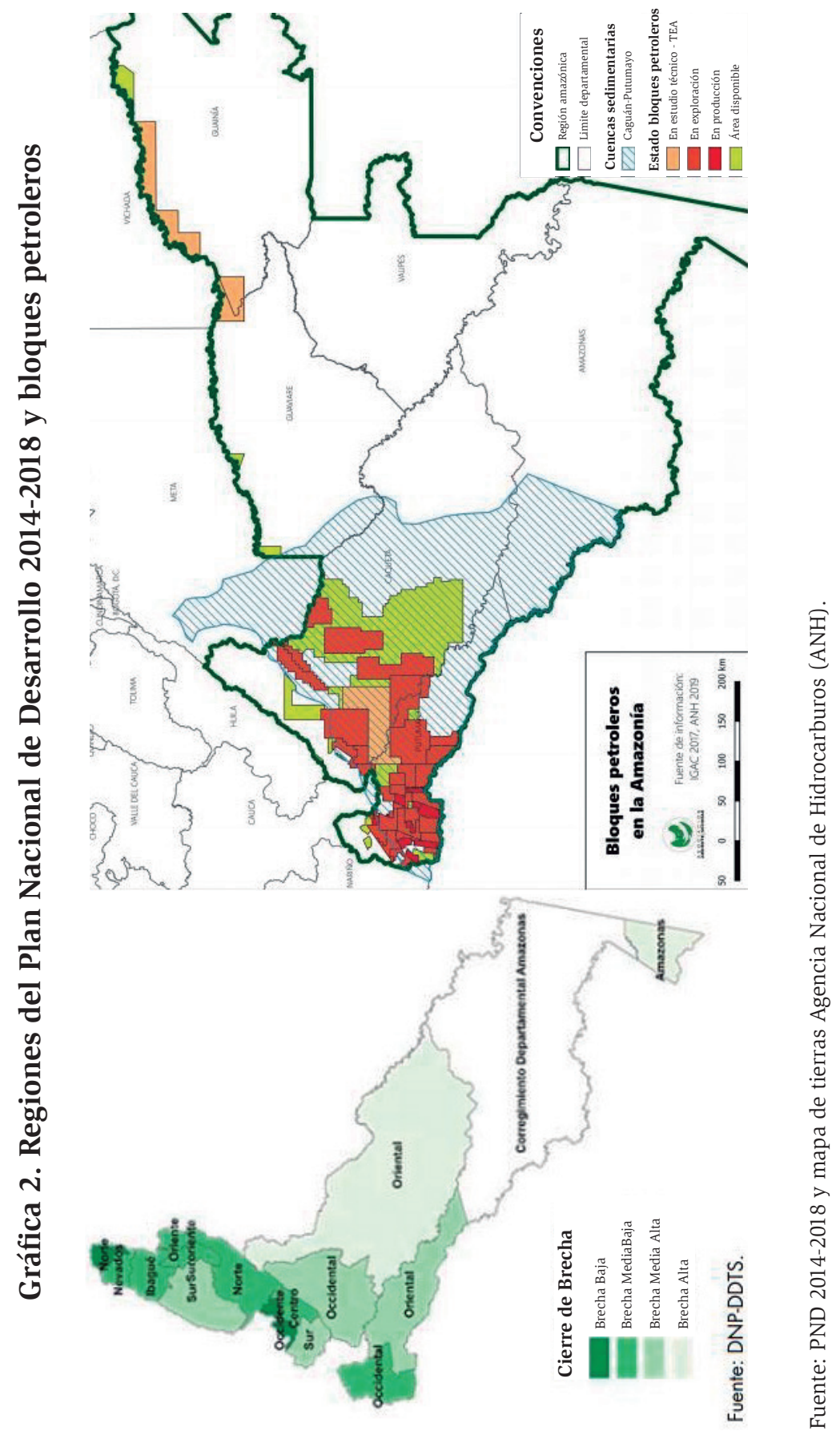


De esta forma, el estado se comporta como el garante de los intereses del capital, brinda garantías jurídicas y contractuales que configuran un nuevo orden institucional en función de los intereses corporativos (Sassen, 2010), al tiempo que designa como opositores del desarrollo a aquellos que exigen seguridad jurídica en torno a la propiedad de la tierra y garantías de retorno. Domingo Emilio Pérez, exalcalde de San Vicente del Caguán expresa

Vemos cómo la empresa entra a chocar fuertemente por la tenencia de la tierra, así como va esto, va para una guerra porque el atropello es grande. Desgraciadamente tenemos un gobierno que nos somete, nos somete a vivir con ellos así nosotros no queramos. Miramos el atraso tan grande, en donde el 50 \% del departamento está adjudicado a estas compañías, mientras nosotros los campesinos no tenemos títulos de tierra, entonces el Gobierno aprovecha esa coyuntura diciendo que como no está titulado, nosotros no somos propietarios de la tierra, solo somos propietarios de las mejoras y que por ello las compañías pueden entrar. (Comunicación personal, 9 de enero del 2015)

Lo anterior se evidencia en el desajuste en el procedimiento de formalización, legalización y adjudicación de tierras a campesinos y las adjudicaciones petroleras, pues según datos del Incoder entre 2004 y 2014 se titularon 194521 hectáreas a campesinos, mientras se adjudicaron 2297886 hectáreas para explotación petrolera en el departamento del Caquetá. Es decir, en términos del acceso a tierras, el 7,8 \% ha sido destinado a población sin tierra, mientras el resto ha sido destinado a adjudicación petrolera. Al respecto, el Instituto SINCHI ha manifestado que

la viabilidad técnica de proyectos de hidrocarburos e hidroeléctricos en zona de reserva forestal, constituyen un tema sensible para las comunidades, pues la sustracción de zonas de reserva para su desarrollo, muestra al Estado como un agente contradictorio frente a los propósitos de conservación. Frente a esto, las comunidades que hacen presencia en la ZRF, esperan que los acuerdos y ejercicios de concertación estén orientados al 
mejoramiento de su calidad de vida y no a su deterioro, toda vez que las circunstancias de su ocupación, en algunos casos, son consecuencia de condiciones estructurales inequidad en cuanto al acceso a mejores oportunidades laborales y de calidad de vida. (SINCHI, 2011, p. 50)

El estado no es, pues, un actor ausente en el Caquetá, por el contrario, como lo plantea Margarita Serje (2011) y el CNMH (2017), la relación entre las instituciones del estado central y el Caquetá ha estado mediada por las lógicas de la dominación militar de lo «salvaje» y «bárbaro»; por lo tanto, su integración a la nación está dada por su funcionalidad como territorio de economías de enclave.

las regiones más alejadas de los ejes concentradores de esa periferia capitalista, se convierten en extrema periferia, en donde el capitalismo salvaje alcanza su máximo poder destructivo. En esa extrema periferia, el capital nacional o internacional trata de obtener el máximo beneficio hacia afuera tratando de fijar el mínimo de la riqueza obtenida en la valorización socioeconómica en el espacio donde trabaja. (Domínguez, 1990, p. 12)

En definitiva, el juego asimétrico que se desprende de la promoción del modelo petrolero como modelo de desarrollo, dentro de un modelo de informalidad y despojo, lleva a la usurpación de derechos de la población que históricamente ha habitado el territorio y las víctimas del conflicto, ante el poder de las empresas petroleras de modelar las formas de intervención del estado a su favor.

\section{Conclusión}

La mirada de enclave extractivo sobre la Amazonía, como zona de frontera y margen de la idea de nación (Serje, 2011), sigue siendo una constante en las políticas de intervención de este territorio. Primero la quina y el caucho, y ahora el petróleo son estrategias de desarrollo con las que muy pocos resultan beneficiados y con las que, en cambio, se instrumentaliza y vende la naturaleza al mejor postor. 
Esta mirada sobre el territorio ha sido promovida y perpetuada por medio de la consolidación de una estructura de tenencia informal y desigual gracias, en buena medida, a la violencia y a la priorización de agendas privadas por encima de los derechos de la población colona y la población víctima del conflicto armado.

En este sentido, vale la pena recordar que los procesos de colonización estuvieron caracterizados por diversas oleadas migratorias propiciadas, principalmente, por la violencia o la falta de oportunidades laborales y de acceso a tierra en el centro del país durante el siglo XX. Muchos campesinos sin tierra vieron en la selva amazónica una oportunidad para mejorar sus condiciones de vida; no obstante, los proyectos frustrados de reforma agraria, el auge de la violencia y ahora, la competencia asimétrica por la tierra con empresas extranjeras, siguen perpetuando su condición de marginalidad.

Esta situación de inequidad, informalidad y violencia es funcional a los intereses de las empresas petroleras que necesitan de espacios «vacíos» para depositar sus excedentes de sobreacumulación. No obstante, este vacío no es natural, sino creado a partir de prácticas de invisibilización y subordinación de personas, sus culturas y formas de relacionamiento distintas con la naturaleza, por medio del desconocimiento de la ocupación en los mapas oficiales y la negación de la región Amazónica como un espacio en el que se construyen identidades.

El caso del municipio de San Vicente del Caguán es representativo de la invisibilización de las personas, pues existe un desfase entre la información oficial del Instituto Agustín Condazzi (IGAC) y la información recopilada por Moreno (2015) y Díaz (2018) sobre el número de veredas del municipio. Según el IGAC el número de veredas es de 152, según Moreno son 344 y según Díaz son 352. Una diferencia tan grande entre la información oficial y estudios realizados en la zona, muestran el tamaño del desconocimiento del territorio por parte de las instituciones centrales y la forma en la que la cartografía del estado niega la ocupación del territorio. 
En el Caquetá, la idea de un espacio vacío surge de la falta de reconocimiento de los predios abandonados por la población desplazada y el desconocimiento de la ocupación por parte de población campesina e indígena que no goza de títulos de propiedad. Para el caso, se evidenció que la Ley 160 de 1994 reforzó los procesos de concentración de la propiedad y ha implicado una disminución de los procesos de formalización de la propiedad rural.

Las estrategias de retoma militar materializadas en el plan Patriota y la puesta en marcha de la PCNT ocasionaron un aumento en las dinámicas de desplazamiento y despojo, con un atenuante: la alta informalidad en la tenencia de la tierra no ha permitido el reconocimiento de la totalidad de los predios abandonados. Así, se evidencia lo planteado por el CNMH (2017) cuando afirma que

Los estudios sobre conflicto armado que indican que los planes de consolidación implicaron un aumento de los conflictos en los territorios resultan ser acertados para el departamento (...) y que las dinámicas de concentración de la propiedad no se deben a la presencia de un actor armado como las FARC, sino a la presencia de confrontaciones entre varios actores armados. (p. 241)

De esta forma, la puesta en marcha de la política de seguridad democrática en el 2002 y la política rural vía regulación del mercado han funcionado como mecanismos de vaciamiento, siendo el estado un actor determinante en este proceso, de ahí que se confirme la hipótesis de Saskia Sassen (2010), según la cual nos enfrentamos a la configuración de un nuevo orden institucional privado que, para el caso de las empresas extractivas, brinda todas las garantías de seguridad física y una ágil sustracción de zonas de reserva forestal y derechos sobre la tierra.

Se confirma también lo planteado por Vargas (2013) con respecto a la relación entre la presencia de industrias extractivas y los problemas agrarios en el Caquetá 
Puede afirmarse que la presencia de complejos mineros en zonas rurales agrava los históricos problemas agrarios, puesto que afecta al campesinado y sus expectativas de adquirir tierras como sujeto de especial protección constitucional, ya que la presencia minera compite territorialmente con actividades agropecuarias (usos del suelo) al requerir acceso a la tierra y otros recursos naturales como el agua para la explotación del subsuelo, o convertirse en un gravamen o limitación a la propiedad, estableciendo obligaciones como las de imponer servidumbres funcionales a la actividad minera. Ello facilita o promueve la acumulación de tierras, el englobe de pequeñas propiedades agrarias, la transformación de usos productivos del suelo, la concentración de la propiedad rural en contra de sujetos de reforma agraria y la especulación en el mercado de tierras. (pp. 59-60)

De todo esto resulta un espacio y tipos de actores marginados, cuyas demandas de retorno y reconocimiento de derechos de propiedad son silenciadas, lo que da lugar a un nuevo ciclo de victimización de población desplazada y tenedores informales, que se agravaría si continúa la rápida adjudicación de tierra a empresas petroleras.

Una de las cuestiones que escapa a este análisis y que debe ser retomada con detenimiento es la tensión entre la ocupación extractiva y las dinámicas de restitución y cumplimiento del punto uno sobre Reforma Rural Integral del Acuerdo de Paz.

Al respecto, la tajante negativa del expresidente Juan Manuel Santos de negociar el modelo económico y la profundización del modelo extractivo en el actual gobierno de Iván Duque, así como la débil agenda que este tiene en el cumplimiento de los Acuerdos —entre ellos los relacionados con las garantías de restitución a las víctimas y los planes necesarios para aclarar la situación jurídica de la tierra (catastro multipropósito y programas de formalización) - plantean un panorama en el que se consolida la perpetuación de una condición de desplazamiento, configurándose un despojo institucional en favor de la industria petrolera. 


\section{Referencias}

Arcila, Oscar (2011). La Amazonía Colombiana Urbanizada: Un análisis de los asentamientos humanos. Bogotá: Instituto Amazónico de Investigación Científica [SINCHI].

Artunduaga, Félix (1987). Historia General del Caquetá. Florencia: Alcaldía Municipal de Florencia, Lotería del Caquetá y Consejo Municipal de Doncello.

Balcázar, Álvaro; López Nelson; Orozco, Martha y Vega, Margarita (septiembre, 2001). Colombia: alcances y lecciones de su experiencia en reforma agraria. Serie Desarrollo Productivo, 109, 5-50. Recuperado de https://bit. ly/2ZaItaZ.

Bolívar, Ingrid (2011). Prácticas disciplinares y promesas de la etnografía: redescubrir el estado. En Chávez, Margarita (Comp.), La multiculturalidad estatalizada. Indígenas, afrodescendientes y configuraciones del estado. Bogotá: Instituto Colombiano de Antropología e Historia [ICANH].

Centro Nacional de Memoria Histórica [CNMH] (2017). La tierra no basta. Colonización, baldíos, conflicto y organizaciones sociales en el Caquetá [informe]. Bogotá: CNMH.

Ciro, Alejandra (2013). "Unos grises muy berracos". Poder Político Local y Configuración del Estado en el Caquetá. 1980-2006 [Tesis de maestría]. Bogotá, D. C.: Universidad Nacional de Colombia.

Consultoría para los Derechos Humanos y el Desplazamientos [CODHES] (marzo, 2012). Desplazamiento creciente y crisis humanitaria invisibilizada [Boletín n. ${ }^{\circ}$ 79]. Bogotá; Quito: CODHES. Recuperado de: https://bit. ly/2KOUmhH.

Corporación para el Desarrollo Sostenible del Sur de la Amazonía [Corpoamazonía] (7 de noviembre del 2012). Reservas Forestal de la Amazonía de la Ley 2 da de 1959. Corpoamazonía. Recuperado de: https://bit.ly/2z8HkG8.

Cubides, Fernando; Olaya, Ana; Ortiz, Carlos (1998). Conclusiones. En Gonzáles, Olga (coord.), La Violencia y el municipio colombiano (pp. 237-244). Bogotá: Universidad Nacional de Colombia. 
Departamento Nacional de Planeación [DNP] (2011). III Crecimiento sostenible y competitividad. En Plan Nacional de Desarrollo 2010-2014. Bogotá: DNP. Recuperado de https://bit.ly/2N7Y7Sd.

Departamento Nacional de Planeación [DNP] (2019) Bases del Plan Nacional de Desarrollo 2018-2022 "Pacto por Colombia, pacto por la equidad", Bogotá: DNP. Recuperado de: https://n9.cl/7q6g

Díaz, Karla (2018). Autogestión y Desarrollo. Tensiones narrativas en torno a la planeación participativa en San Vicente del Caguán, Colombia, 2011-2017 [Tesis de Maestría]. México: Colegio de la Frontera Norte.

Domínguez, Camilo (1990). Economía extractiva en la Amazonía colombiana 1850-1930. Bogotá: Tropenbos-Corporación Araracuara.

Faivre, Hortense (2005). Del Plan Colombia al Plan Patriota: Impacto del conflicto armado colombiano en el Ecuador, análisis de una estrategia de seguridad subregional. Revista Complutense de Historia de América, 31, 215230. Recuperado de https://bit.ly/2Nb39gM.

Fundación Ideas para la Paz [FIP] (septiembre, 2011). Balance de la Política Nacional de Consolidación Territorial [Informe n. ${ }^{\circ}$ 14]. Bogotá. Recuperado de: https://bit.ly/2m06SC6.

Garavito, Aarón; Iregui, Ana María y Ramírez Teresa (2012). Inversión extranjera en Colombia: Evolución reciente y marco normativo. Borradores de Economía, 713, 1-63.

Gómez, Rosana (junio, 2017). Caracterización del Medio Físico-Biótico. En Actualización POMCA Rio Hacha, fase diagnóstica. Corpoamazonía.

González Camilo (marzo, 2012). Consolidación territorial, y resurgimiento de paras y guerrilla [Informe]. Bogotá. Recuperado de https://bit.ly/2OZOFEB.

Harvey, David (2004). El “nuevo” imperialismo: acumulación por desposesión. Socialist Register, 40, 99-125 Recuperado de https://bit.ly/2Z7wrPK.

Hormaza, Ingrid (2016). La reforma agraria como ejercicio de planificación, experiencia de los proyectos de colonización del INCORA en el Caquetá entre 1964-1974 [Tesis de Maestría], Bogotá D.C.: UNAM. 
Instituto Amazónico de Investigaciones Científicas SINCHI (2011). Zonificación ambiental y ordenamiento de la reserva forestal de la Amazonia, creada mediante la Ley $2^{a}$ de 1959, en los departamentos de Caquetá y Huila. [Informe final]. En versión 2.0, del convenio 016 de 2010. Bogotá, D. C.: Grupo de Gestión de Información Ambiental y Zonificación del Territorio: Amazonia Colombiana [GIAZT].

Instituto Geográfico Agustín Codazzi [IGAC] (2014). Sistema de Información para la planeación y el ordenamiento territorial SIGOT) [portal web]. Recuperado de http://sigotn.igac.gov.co/sigotn/.

Martínez, Sandra (2016). Más allá de la gubernamentalidad: políticas de colonización y desarrollo rural en el piedemonte caqueteño (1960-1980), Universitas Humanística, 82, 135-162.

Millet, Damien y Toussaint, Éric (2005). Deuda Externa, Banco Mundial y FMI. 50 preguntas/50 respuestas. Quito: Ediciones Abya-Yala.

Mondragón, Héctor (2001). Dónde está la tierra y cómo remediarla. En Ministerio de Agricultura y Desarrollo Rural [MADR], Colombia, tierra y paz. Experiencias y caminos para la reforma agraria. Alternativas para el siglo XXI. Bogotá: MADR.

Moreno, Diana (2015). "El estado somos nosotros": prácticas organizativas comunitarias de la zona rural de San Vicente del Caguán-Caquetá, como materialización de la construcción de estado local en Colombia [Tesis de Maestría] Bogotá: Universidad de los Andes.

Neva, Nelson (2014). Bases conceptuales procesos de regularización de la propiedad rural y acceso a tierras. Bogotá: UPRA.

Organización de las Naciones Unidas para la Alimentación y la Agricultura [FAO] (2003). "Qué es la tenencia de la tierra”. En Tenencia de la tierra y desarrollo rural. Roma: FAO.

Pardo, Alvaro y Duarte, Javier (abril, 2018). ¿Y si se eliminan los beneficios al sector extractivo?, Justicia Tributaria e Institute of International Education. Recuperado de https://bit.ly/31Le4la.

Peña, Rocío; Parada, María; Zuleta, Santiago (enero-junio, 2014). La regulación agraria en Colombia o el eterno déjà vu hacia la concentración y el despojo: un análisis de las normas jurídicas colombianas sobre el agro (19912010). Revista Estudios Socio Jurídicos, 16(1), 121-164. 
Polanyi, Karl (2007). La Gran Transformación. Crítica del liberalismo económico. Madrid: Quiupu Editorial.

Presidencia de la República. Colombia (14 de noviembre de 2018). Presidente Duque destaca aportes de la industria petrolera al desarrollo del país. Presidencia de la República. Recuperado de: https://bit.ly/2KFV1TR.

Ramírez, Henry (3 de septiembre de 2009). ¿Bases Militares en Colombia, extensión de acuerdos militares o implementación de la Escuela de las Américas? En Comisión Intereclesial de Paz y Justicia. Recuperado de https:// bit.ly/2m8cwCo.

Registro Nacional de Áreas Protegidas [RUNAP] (s.f.). Departamento del Caquetá, disponible en: http://runap.parquesnacionales.gov.co/departamento/939.

Reyes, Alejandro (2009). Violencia, conflictos agrarios y poder en las regiones colombianas. En Guerreros y Campesinos. El despojo de la tierra en Colombia, pp. 15-55. Bogotá: Norma.

Rodríguez, Ivonne (2013). Despojo, baldíos y conflicto armado en Puerto Gaitán y Mapiripán (Meta, Colombia) entre 1980 y 2010, Estudios socio-jurídi$\cos 16(1), 315-342$.

Sassen, Saskia (2010). Nueva geografía política. Un nuevo campo transfronterizo para actores públicos y privados. Texto de la conferencia del Millenium, en London School of Economics, el 25 de enero de 2000, retomado de la conferencia inaugural de la cátedra de ciencias sociales en la Universidad de Chicago, el 28 de abril de 1999. Recuperado de https://bit.ly/2Zbd7Vz.

Serje, Margarita (2011). El revés de la Nación: Territorios salvajes, fronteras y tierras de nadie. Bogotá: Universidad de los Andes.

Unidad para la Atención y Reparación Integral a las Víctimas [UARIV] ( $1^{\circ}$ de noviembre del 2018). Desplazamiento, personas. Recuperado de https:// cifras.unidadvictimas.gov.co/Home/Desplazamiento.

Unidad de Planificación Rural Agropecuaria [UPRA] (2017). Índice de informalidad. Bogotá: UPRA. Recuperado de: https://bit.ly/2FUPjtL. 
Unidad de Planificación Rural Agropecuaria [UPRA (2015). Socialización de resultados técnicos UPRA [jornada de inducción y reinducción]. Bogotá: UPRA. Recuperado de: https://bit.ly/2DM7BfA.

Vargas, Fernando (2013). Minería, Conflicto armado y despojo de tierras: Impactos, desafíos y posibles soluciones jurídicas. En Garay, Jorge (Comp.), Minería en Colombia. Fundamentos para superar el modelo extractivista, pp. 9-19. Bogotá: Contraloría General de la República. 